\title{
(Ultra) Structural Details of Cells and Matrix during Early Cartilage Regeneration after Chondrocyte Implantation in the Equine Knee Joint
}

\author{
Nurnberger Sylvia ${ }^{1,2,3 *}$, Barnewitz Dirk Dvm ${ }^{4}$, Tichy Brigitte ${ }^{1}$, Ponomarev Igor ${ }^{4}$, Meyer Christof ${ }^{5,6}$, Klepal W$^{7}$, Albrecht Christian $^{1,3}$ and \\ Marlovits Stefan ${ }^{1,3}$
}

${ }^{1}$ Department of Trauma Surgery, Medical University of Vienna, Währinger Gürtel, 18-20, 1090 Vienna, Austria

${ }^{2}$ Ludwig Boltzmann Institute for Experimental and Clinical Traumatology in AUVA Research Center, Austrian Cluster for Tissue Regeneration, Donaueschingenstrasse 13, 1200 Vienna, Austria

${ }^{3}$ Bernhard Gottlieb University Clinic of Dentistry Sensengasse 2a 1090 Vienna, Austria

${ }^{4}$ Research Centre for Medical Techniques and Biotechnology, Geranienweg 7, 99947 Bad Langensalza, Germany

${ }^{5}$ University of Giessen, Clinic and Polyclinic for Traumatology, Rudolf-Buchheim-Straße 7, 35385 Giessen, Germany

${ }^{6}$ Orthopaedic and Trauma Surgery, Klinikum Saarbrücken, Winterberg 1, 66119 Saarbrücken, Germany

${ }^{7}$ University of Vienna, Faculty of Life Science, Core Facility Cell Imaging and Ultrastructure Research, Althanstrasse 14, 1090 Vienna, Austria

\begin{abstract}
Objective: This pilot study of cartilage defect treatment was designed to establish the horse as a suitable animal model for MACT (Matrix-Associated Autologous Chrondrocyte Transplantation) transplants and to analyse the morphological aspects of repair tissue development and differentiation.

Design: Hyaluronan-based and collagen-based biodegradable scaffolds were seeded with autologous chondrocytes and implanted into large $(1.5 \times 2 \mathrm{~cm})$ defects in the trochlear ridge of the distal femur of three horses. A non-treated, empty defect was used as control. Three months after surgery, samples of the defect area were investigated using bright field and polarized light microscopy, immunohistochemistry and electron microscopy.

Results: In MACT-treated lesions with good defect filling, the repair tissue integrated well into the defect and showed features of differentiation in transition to native cartilage; the matrix was partially masked by proteoglycans, strongly stained for collagen type II, and the fibres had the typical vertical arrangement of articular cartilage. In areas with less intense staining, collagen type II formed a network around the cells. Calcified cartilage was partially decalcified and osteoclasts as well as osteoblasts reorganised the subchondral bone. The control defect was almost empty.

Conclusion: This study showed that the horse is a suitable animal model for MACT. Differentiation starts early after transplantation in the periphery of the defect. Collagen type II precedes proteoglycan deposition and undergoes a kind of vertical self-arrangement which is hypothesized to develop from a fibre network around the chondrocytes.
\end{abstract}

Keywords: Cartilage regeneration; Collagen arrangement; Matrix maturation; Tissue engineering; Ultrastructure;Immunohistochemistry; Polarized light microscopy

Abbreviations: CC: Calcified Cartilage; ECM: Extracellular Matrix; NC: Native Cartilage; RT: Repair Tissue; SB: Subchondral Bone; SP: Subchondral Plate

\section{Introduction}

In the treatment of traumatic articular cartilage defects, clinical science presently focuses on long-lasting tissue engineering approaches such as "Matrix-associated Autologous Chondrocyte Transplantation" (MACT). During this procedure the patient's own (autologous) chondrocytes are expanded in monolayer cultures, seeded on a 3D carrier (scaffold) and reinserted into a defect [1].

Despite generally favourable clinical outcomes, a certain amount of transplant failures have been observed, the mechanisms of which are not fully understood. Therefore, in vivo investigations in an appropriate animal model have been suggested to help clarify open questions in the postoperative development of the transplants and the Repair Tissue (RT) [2].

The horse is generally considered to be a good animal model for cartilage defect treatment because joint dimension as well as cartilage morphology and physiology are similar to that of humans and because horses suffer from the same degenerative and traumatic injuries [3-5]. A detailed characterisation of equine chondrocytes on two structurally very different MACT scaffolds has recently been published [6] (Figure
1). The study showed that the cells reacted well and in a similar way to human chondrocytes on these materials, and that the distribution of the chondrocytes strongly depended on the material density. One aim of the present study was to demonstrate that soft MACT scaffolds can indeed be applied to horses in vivo using the same surgical procedure as in humans, without postoperative complications (e.g. reaction to the scaffold) and that the transplants are able to give rise to RT. Furthermore, we used the animal model to analyse the morphology of the early (three month) transplant-derived RT development as has previously been done with other cartilage regeneration methods in the horse [7-9]. In particular, we focused on matrix architecture. This is of special interest because matrix architecture and development have been studied mainly in Native Cartilage (NC) and during ontogenesis, but

*Corresponding author: Nurnberger Sylvia, Department of Traumatology, Medical University of Vienna, Währinger Gürtel 18-20, 1090 Vienna, Austria, Tel: +43 140400 5944; Fax: +43 140400 5947; E-mail: sylvia.nuernberger@meduniwien.ac.at

Received April 04, 2013; Accepted December 12, 2013; Published December 14,2013

Citation: Sylvia N, Dirk B, Brigitte T, Igor P, Christof M, et al. (2013) (Ultra) Structural Details of Cells and Matrix during Early Cartilage Regeneration after Chondrocyte Implantation in the Equine Knee Joint. J Tissue Sci Eng S2: 004 doi:10.4172/2157-7552.S2-004

Copyright: ( 2013 Sylvia N, et al. This is an open-access article distributed under the terms of the Creative Commons Attribution License, which permits unrestricted use, distribution, and reproduction in any medium, provided the original author and source are credited. 


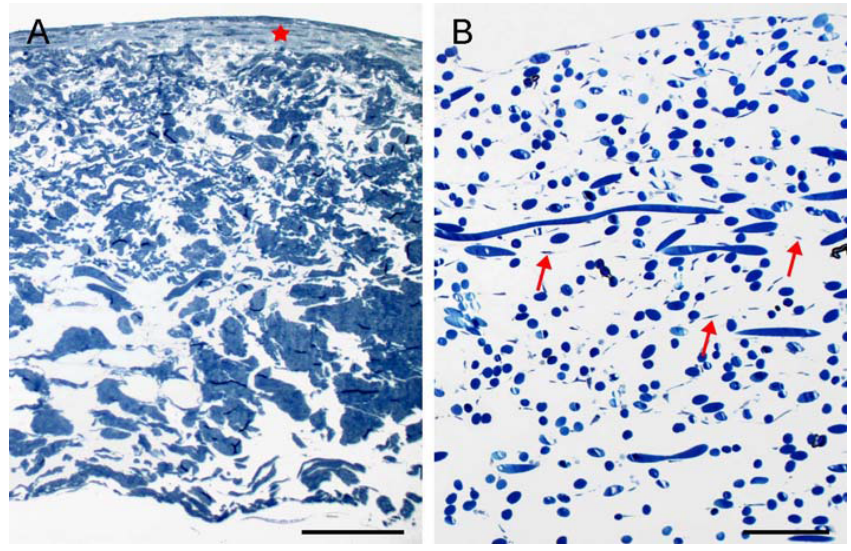

Figure 1: The two scaffold types used as carrier for chondrocyte transplantation in this project showing very different structure and cell distribution. A) A dense collagen type I/III fleece consisting of collagen fibres of various thickness. Most of the chondrocytes are on the seeding surface of the material forming a cellmultilayer (asterisk). B) Loose hyaluronan web of three-dimensionally arranged scaffold fibres with regular thickness. The cells are distributed uniformly between the large interfibre spaces (arrow). Scale bars: (A,B) $100 \mu \mathrm{m}$. (For further description see Nernberger et al. 2012)

little is known about the process in the context of regenerating articular cartilage tissue $[10,11]$.

\section{Material and Methods}

\section{Animal model}

Four healthy yearling mares that did not meet breeding criteria were obtained from the Haflinger Stud Meura, Thüringen, Germany. During the entire study the animals were kept and treated at the horse clinic of the Research Centre of Medical Technology and Biotechnology (fzmb GmbH) in Bad Langensalza, Thüringen, Germany, under the surveillance of veterinarians. The study was verified and approved by the local ethical board (Thüringer Landesamt für Lebensmittelsicherheit und Verbraucherschutz Nr. 14-03/03).

\section{Transplant generation}

Chondrocytes were harvested from cartilage chips (1-2 g) taken from the talocrural joints of all horses except for the control animal. All the following steps were performed for each biopsy/horse separately as described in detail in Nürnberger et al. [6]. Chondrocytes were enzymatically isolated, seeded in culture flasks and passaged when confluent. In passage two, chondrocytes were transferred to two structurally very different biodegradable carrier materials:1) the loose hyaluronan web (HYAFF ${ }^{\circledR}$; Fidia, FAB, Italy) and 2) the dense collagen type I/III fleece (BioGide ${ }^{\circledR}$, Geistlich Pharma, Switzerland) at a concentration of $10^{6} \mathrm{cells} / \mathrm{cm}^{2}$ and cultivated for a further three weeks.

\section{Surgery}

Three horses were treated with transplants eight weeks after the biopsy. Anterolateral arthrotomy of the left knee joint was performed with the horses in dorsal recumbency. A $1.5 \times 2 \mathrm{~cm}$ defect was set in the trochlear ridge of the distal femur without damaging the Subchondral Plate (SP; consists of calcified cartilage plus subchondral bone). Two horses were treated with one transplant each, either the hyaluronan web or the collagen fleece. The third animal was treated with both transplants (one of each transplant type) in a double sandwich-like layer with the hyaluronan web facing the SP and the collagen fleece on top. Fibrin glue (Beriplast ${ }^{\circledR}$ P, Combi-Set $3 \mathrm{ml}, \mathrm{Fa}$. Aventis Behring, Marburg) was applied at the interface of transplants to the host cartilage. The stability of the graft in the defect was tested by passive movement of the leg over the entire range of motion. In the control animal, the same defect was created but not treated with transplants. All surgeries were carried out without complications. Horses were kept for two weeks in boxes, then exercised (walked) under controlled conditions for 30 days and thereafter kept in paddocks. Postoperative follow-up care was also without complications.

\section{Sampling}

Three months after implantation, the horses were euthanized and the defect area prepared for taking overview images and further processing. Tissue sections were taken from the interface regions and the centre of the defect. Sections were fixed with $2.5 \%$ glutaraldehyde in $0.05 \mathrm{M}$ sodium cacodylate ( $\mathrm{pH} 7.3$ ), rinsed, photographed and postfixed with $0.5 \%$ osmium tetroxide containing $1 \%$ potassium ferrocyanide. Then they were dehydrated in a gradient series of alcohol and embedded in Agar100 resin via propylene oxide. Semithin sections $(1 \mu \mathrm{m})$ and ultrathin sections $(70 \mathrm{~nm})$ were sequentially cut. The former were either stained with toluidine blue or deplastified and used for immunohistochemical reaction against a monoclonal collagen type II antibody (Neomarkers, CA, USA) and visualized with diaminobenzidine tetrahydrochloride and haematoxylin counterstaining. Ultrathin sections were stained with uranyl acetate and lead citrate. Alternatively, some tissue sections were fixed with $7.5 \%$ formaldehyde, dehydrated, blocked in paraffin and sectioned for immunohistochemical reaction as described above. Light microscopic sections were examined under a Nikon Eclipse E800 and Olympus BX41 with polarization filter for collagen fibre orientation. Ultrastructural investigations were done at a Zeiss 902 (Oberkochen, $\mathrm{DE}$ ) and FEI Morgagni electron microscope (Eindhofen, NL).

\section{Results}

\section{Gross anatomy}

In the macroscopic view, the surfaces of the three transplanted defects were totally covered by RT (Figure 2A and F). The control defect contained little tissue which only sparsely covered the CC at some sites (Figure $2 \mathrm{~K}$ ). In two defects treated with a single transplant layer, RT reached the level of NC and strongly resembled the whitish $\mathrm{NC}$ in appearance (Figure 2A-2D). In contrast, the defect treated with a double layer transplant was subtotally filled (Figure 2F-2I) suggesting that the upper layer was pulled out of the defect in the course of the postoperative period. In this case, the RT in the defect was somewhat translucent and appeared slightly reddish in top view because of the subjacent bone (Figure $2 \mathrm{~F}$ ). In all samples the centre of the defect was less dense and less highly filled with RT (about $1 / 2$ to $3 / 4$ of the thickness) than the periphery of the defect. In the control defect, fringy patches of translucent fibrous tissue were loosely attached to the SP (Figure 2L).

\section{Distribution and characteristics of the hyaline-like repair} tissue (RT)

Well-filled defects, treated with one layer of transplant (cell-seeded hyaluronan web or collagen type I/III fleece) showed hyaline-like characteristics in the periphery of the defect. In sections, the tissue stained strongly with toluidine blue and for collagen type II, was homogeneous and contained spherical chondrocytes (Figure 2C-2E). Locally this tissue formed up to two-thirds of the RT-height (Figure 2C and 2D). The hyaline-like matrix was dense, was almost as homogeneous as in NC and merged with it completely, without any obvious edge at the interface (Figure 3A). The fibres, however, were not entirely masked by proteoglycans, making their alignment discernible. The fibres had 

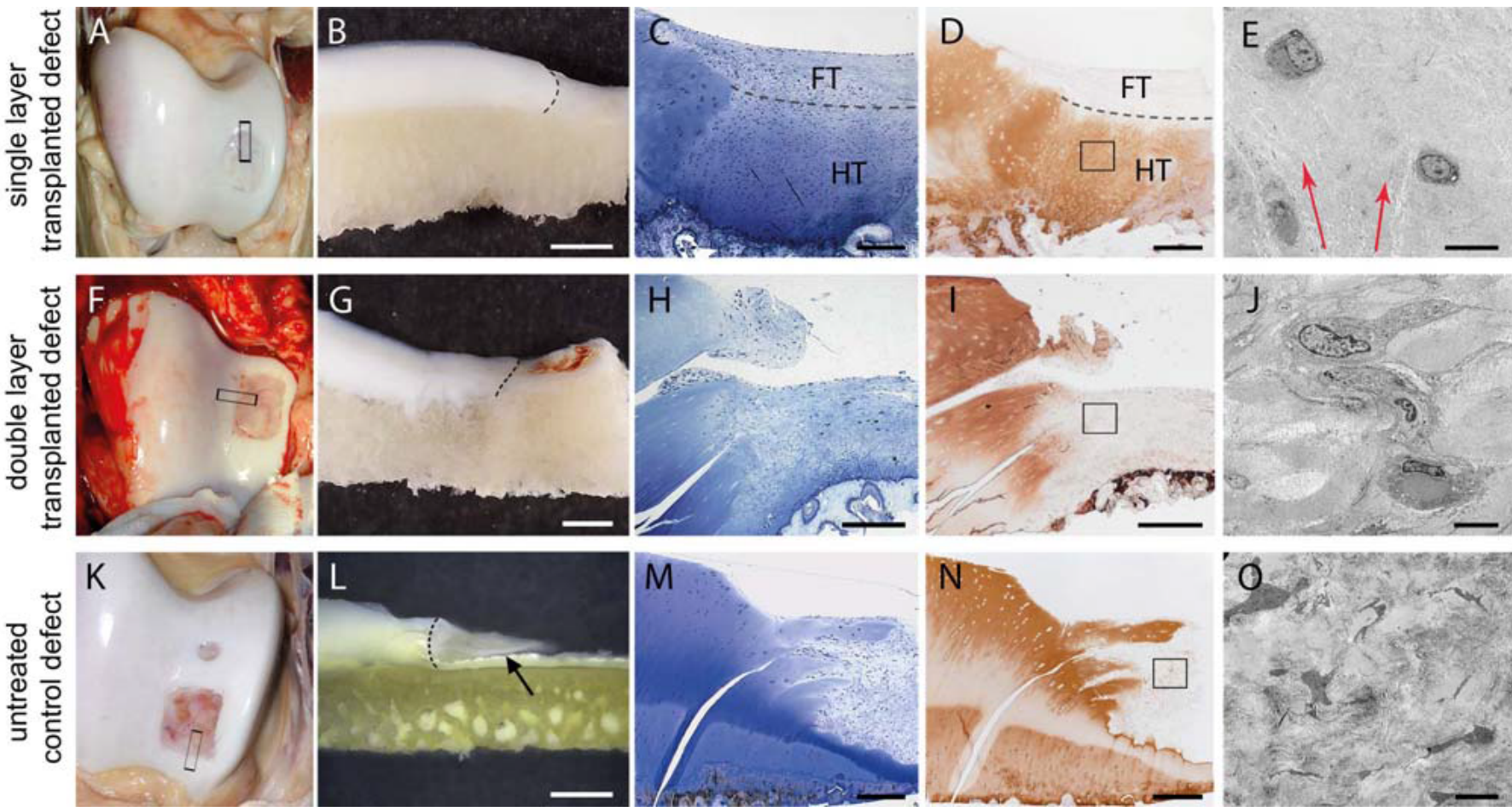

Figure 2: Overview plate of the defects in the trochlear ridge of the distal femur treated with A-E: a single layer (hyaluronan web), F-J: double layer (hyaluronan web \& collagen type I/III fleece) of transplants, and K-O an untreated defect as control. The macroscopic overview images show A: a well-filled defect containing whitish tissue, $\mathrm{F}$ : a defect with translucent tissue and reddish appearance due to the underlying bone in the transplanted defects, and $\mathrm{K}$ : only local tissue in the control. The squares indicate the areas from which the tissue sections in $B, G$ and $L$ were taken. $B, G$ and $L$ : In those sections the complete filling (B) and incomplete filling (G, L) are visible. The dotted line indicates the interface between RT and NC. Only in the control does the RT not adhere well to the calcified cartilage (arrow). In the single transplanted defect C: toluidine blue staining and D: immunohistochemistry against collagen type II (brown staining) show hyaline-like tissue (under the dotted line) similar to NC. In the double transplanted defect and the non-transplanted control, $\mathrm{H}, \mathrm{I}$ and $\mathrm{M}, \mathrm{N}$ the whole RT is pale with little toluidine blue staining ( $\mathrm{H}$, M) and not positive for the collagen type II reaction (I, N). E, J and O: TEM images demonstrating the different structure of matrix and cells. In the hyaline tissue (E), the matrix is homogeneous and only few fibres are visible extending upwards to the joint surface (arrow). The fibres appear bright in the generally grey matrix. In the fibrous tissue of the double transplanted defect $(\mathrm{J})$ and the control $(\mathrm{O})$, the matrix is very fibrous and the cells polygonal. In the control the cells are extremely irregular and dark. Scale bars: (B, G and L) $1 \mathrm{~mm},(\mathrm{C}, \mathrm{D}, \mathrm{H}, \mathrm{I}, \mathrm{M}$ and N) $250 \mu \mathrm{m},(\mathrm{E}, \mathrm{O}) 10 \mu \mathrm{m},(\mathrm{J}) 5 \mu \mathrm{m}$.

the cartilage-specific vertical orientation stretching from the calcified plate towards the cartilage surface, but were slightly wavy (Figure $3 \mathrm{~A}$ and B). Sometimes fibres crossed each other, transverse to the general orientation and appeared to form a network around the chondrocytes (Figure 3C and D). An especially fine network was also present around chondrocytes in matrix with weak collagen type II staining and no vertical fibre strands (Figure $3 \mathrm{E}$ ). At the upper end of the collagen type II positive area, the stained fibres extended into the dedifferentiated region of disorderly arranged, unstained fibres, indicating collagen type I (Figure 3F and G). The control, in comparison, shows the different appearance of collagen type II fibres from fringy cartilage edges stretching into fibrous RT. In these locations, bunches of parallel fibres extended locally from the very dense and homogeneous NC edge (Figure 3H).

Both in the hyaline and the fibrous RT, occasionally dense and thick fibre strands were embedded in the Extracellular Matrix (ECM) (Figure $4 \mathrm{~A}$ and $\mathrm{B}$ ). The morphology and dimension of this material resembled the fibre strands of the collagen type I/III fleece as observed in in vitro investigations (Figure 4C). In the horse study, those structures were found only in the defect treated with this specific transplant type, indicating that they were transplant residuals.

Cell density in the hyaline-like RT was generally higher than in the NC but all cells were clearly spaced apart from each other, with a separation of about one to several cell diameters (Figure 2C, 3A and Figure 5A). Only in double or multichondron-like chondrocyte groups were cells even closer, but a rim of matrix still prevented intercellular contact (Figure 5G). Most chondrocytes embedded in the hyaline-like matrix had the typical characteristics of differentiated chondrocytes, albeit not as well expressed as in NC. Cell shape was spherical (Figure $5 \mathrm{~F}-\mathrm{H}$ ) or somewhat wavy and dented (Figure 5E). The nuclei were typically located peripherally in the cells and were spherical or ovoid. Some of the chondrocytes contained glycogen, lipid droplets and small bundles of intermediary filaments around the nucleus (Figure $4 \mathrm{~A}$ and $5 \mathrm{G})$. A pericellular matrix had sometimes developed (Figure 5G).

\section{Fibrous Repair Tissue (RT)}

With increasing distance to the hyaline-like RT, both matrix as well as cellular characteristics of differentiation became less expressed towards the centre and the surface of the defect. The matrix stained progressively less intensively with toluidine blue, which corresponds to a decrease in proteoglycans (Figure $2 \mathrm{C}$ and $5 \mathrm{~A}$ ). Due to the reduced masking, the matrix appeared more fibrous. The collagen fibres lost their vertical orientation and formed disorderly arranged collagen bundles that did not stain for collagen type II (Figure 2D, 3G, 5A, 6A). With the decrease in the hyaline character of the matrix, the cells formed more cell extensions (Figure 5D). In completely fibrous areas, cells were polygonal with long slender cell projections, which were frequently 
Citation: Sylvia N, Dirk B, Brigitte T, Igor P, Christof M, et al. (2013) (Ultra) Structural Details of Cells and Matrix during Early Cartilage Regeneration after Chondrocyte Implantation in the Equine Knee Joint. J Tissue Sci Eng S2: 004. doi:10.4172/2157-7552.S2-004
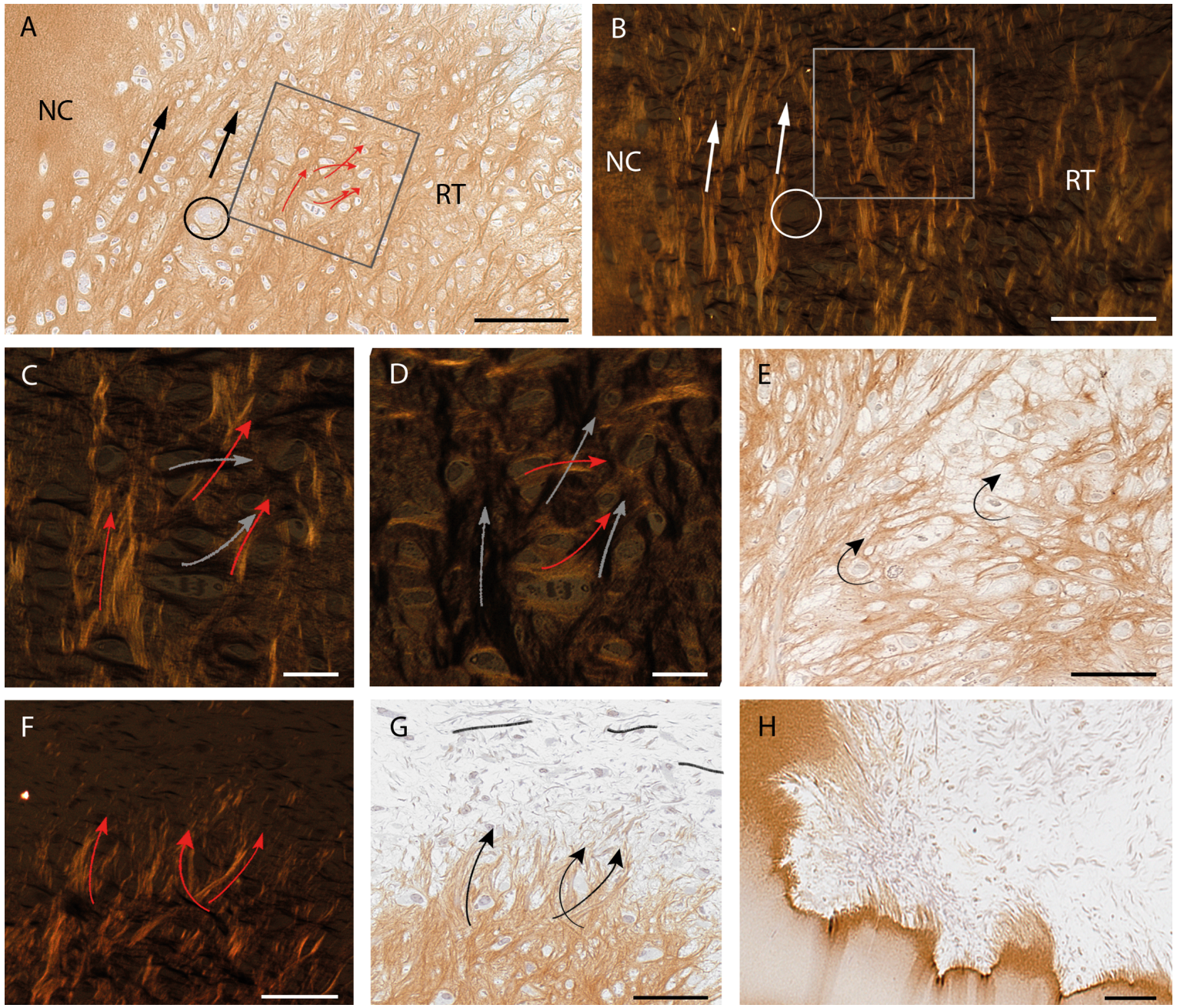

Figure 3: Matrix organisation of the differentiated RT of a defect treated with chondrocytes on a hyaluronan web (A-D, F, G) and collagen fleece (E) in comparison to the control $(\mathrm{H})$. A, E, G and H: Immunohistochemical reaction with collagen type II antibodies and B, C, D and F: Polarized light microscopy. A: The RT shows the same intense staining for collagen type II antibody but a more fibrous appearance than the NC. The fibres are wavy and vertically arranged (large arrows) and occasionally cross each other (small arrows). Note that the cellularity in the RT is higher than in the NC. The circles in A \& B indicate the same areas to show that the images were taken from the same section. The squares in A \& B label the areas shown in high magnification in C \& D. B: In polarized light the vertical fibre arrangement (arrows) is especially well discernible because only fibres with the same orientation appear bright. In addition, oblique and horizontal fibres are visible (appear dark) in between the vertical strands. C, D: Detail images of the matrix in polarized light in two different orientations showing either the vertical or oblique fibres. The arrows correspond to those in (A). E: RT area with weak collagen type Il positive staining forming a network-like feature around the cells (curved arrows). In the left of the image, collagen type II stained fibres are vertically and more densely arranged. F, G: Upper margin of the hyaline-like RT in transition to the fibrous RT, showing vertically arranged, wavy collagen type Il-positive fibres (bent arrows) extending into an area of unstained, three-dimensionally arranged fibres (horizontal lines). H: Periphery of a control defect. Locally, fibre-bunches extend from residual cartilage into the defect area. Scale bars: (A, B) $100 \mu \mathrm{m},(C, \mathrm{D}) 20 \mu \mathrm{m}$, (E-H) $50 \mu \mathrm{m}$
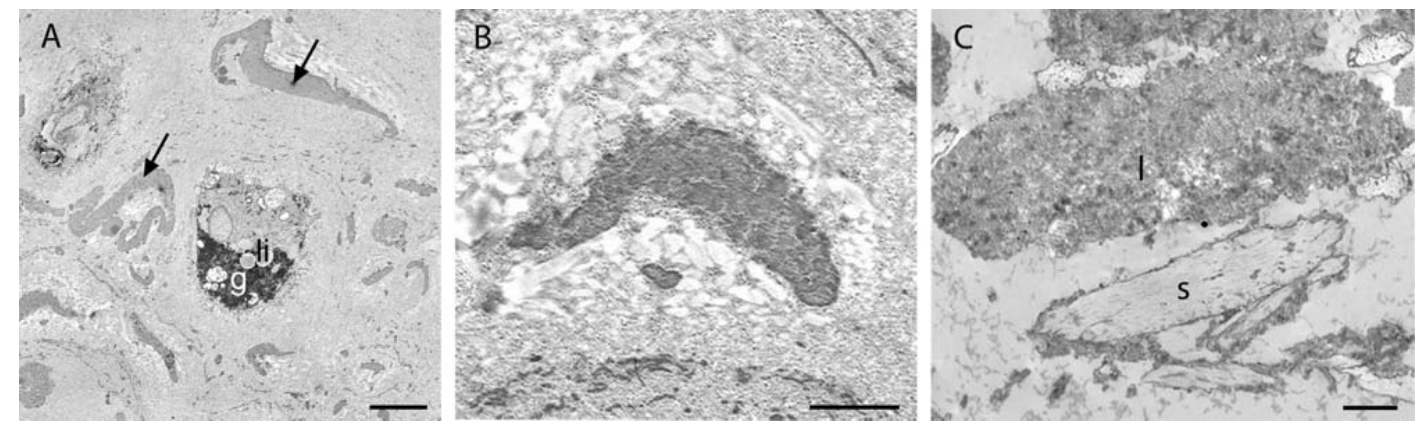

Figure 4: TEM images of a hyaline-like RT area treated with a chondrocytes seeded collagen type I/III fleece showing a differentiated chondrocyte containing glycogen (g, black area) and lipid (li, bright droplets) in the cytoplasm and large, compact fibre strands (arrows) embedded in the dense matrix surrounding the cell (A, B). In high magnification the fibre strands resemble the structure of the scaffold material (collagen type I/III fleece). C: Image of the pure scaffold material collagen type I/III fleece directly taken from cell culture shows large, dark fibres (I) and smaller, brighter ones (s). Scale bars: (A) $5 \mu \mathrm{m}$, (B and C) $2 \mu \mathrm{m}$. 
Citation: Sylvia N, Dirk B, Brigitte T, Igor P, Christof M, et al. (2013) (Ultra) Structural Details of Cells and Matrix during Early Cartilage Regeneration after Chondrocyte Implantation in the Equine Knee Joint. J Tissue Sci Eng S2: 004. doi:10.4172/2157-7552.S2-004

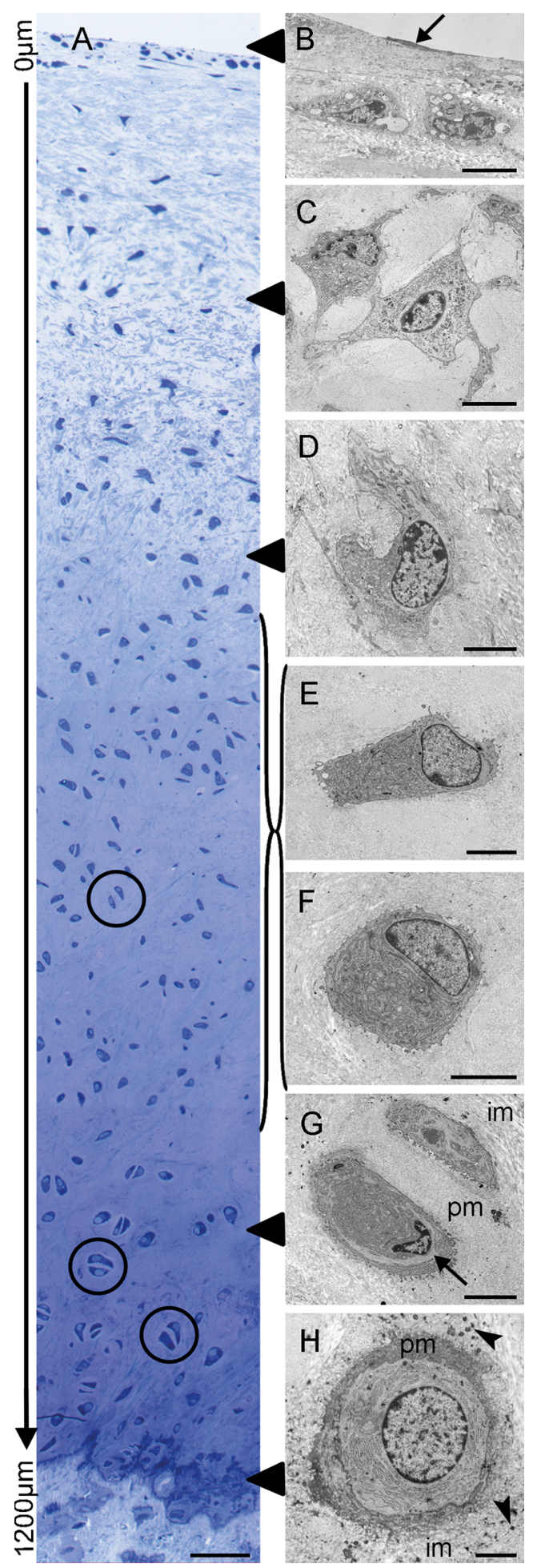

Figure 5: Section through a RT of a cartilage defect treated with chondrocytes on a hyaluronan web (detail of Figure 1C) showing hyaline-like tissue in the lower half and fibrous tissue in the upper half. A: The toluidine blue stained light microscopic image shows homogeneously and densely stained ECM in the lower half and a fibrous, less dense matrix in the upper half of the column. The cells are regularly distributed, either singularly or in pairs (circles). The cellularity is higher in the deeper than in the superficial regions. B-H: TEM images show the alteration of chondrocytes from the surface to the depth of the RT. B: Superficial chondrocytes of different shape, separated by a matrix layer from the joint surface. Note the thin dark sheath locally covering the matrix (arrow). C: Polygonal chondrocytes extending several cell processes into a dense but fibrous matrix. D: Similar polygonal cell as in C but with fewer and thicker cell processes and with a small bundle of intermediate filaments around the nucleus. E, F: Cells in the upper, hyaline-like tissue are by tendency (E) or totally (F) spherical. The matrix is very dense and homogeneous. G: In the deepest region of the hyaline-like RT, many chondrocytes are ovoid and arranged as double chondrons. Note the bundle of intermediar filaments around the nucleus (arrow) and the Pericellular Matrix (pm) between the cells; im=interterritoreal matrix; $\mathrm{H}$ : Chondrocyte close to the tide mark surrounded by a dark Pericellular Matrix (pm) and matrix vesicles (arrowhead) in the Interterritoreal Matrix (im). Scale bars: (A) $50 \mu \mathrm{m},(B-G) 5 \mu \mathrm{m},(\mathrm{H}) 2 \mu \mathrm{m}$. 
Citation: Sylvia N, Dirk B, Brigitte T, Igor P, Christof M, et al. (2013) (Ultra) Structural Details of Cells and Matrix during Early Cartilage Regeneration after Chondrocyte Implantation in the Equine Knee Joint. J Tissue Sci Eng S2: 004. doi:10.4172/2157-7552.S2-004
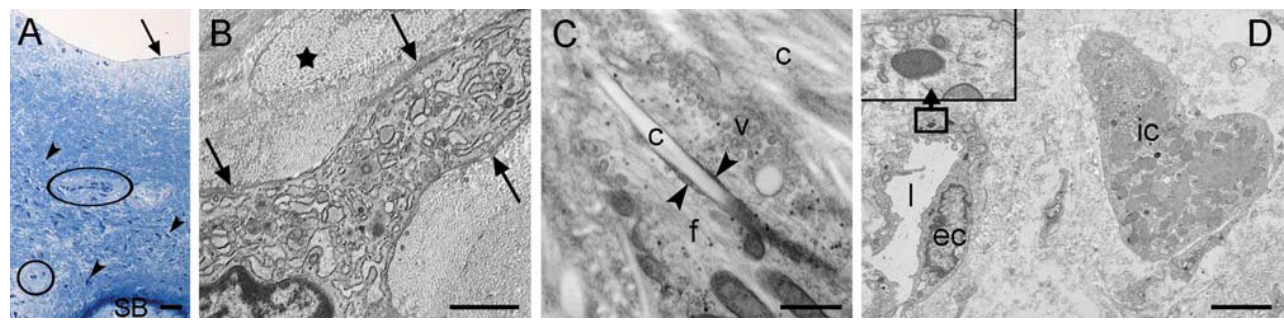

Figure 6: Characteristics of fibrous RT of transplanted cartilage defects shown by toluidine blue stained semithin sections (A) and TEM images (B,C,D). A-D: Fibrous tissue of the middle of the cartilage defect treated with chondrocytes on a double sandwich-like layer of a hyaluronan web and a collagen type I/II fleece. A: The tissue contains vascular-like structure in the lower part of the RT (circles), many cells (dark blue dots; arrowheads) in deep regions and fewer cells in the upper half. The surface is smooth and locally covered by a dark layer (arrow). SB=Subchondral Bone. B: Part of the cell body and cell extensions of a polygonal chondrocyte embedded in fibrous matrix. Note the organisation of collagen in form of strands (asterisk). A very thin rim of electron-dense matrix (arrow) is locally visible along the cell membrane. The cytoplasm of the cell is partially dominated by dilated rER. C: Detail image of a cell extension of a polygonal cell containing loose strands of Filaments (f) in the cytoplasm and many Vesicles ( $v$ ) aligned at and budding from the cell membrane. The large bright fibre in the middle of the cell extension is a Collagen fibril (c) penetrating the cell in a deep invagination (note that it has the same appearance as the collagen fibrils in the matrix surrounding the cell). The fibril is flanked by the cell membrane (arrowheads). D: Vascular-like structure formed by Endothelial Cells (ec; I: Lumen) with dark Weibel-Palade-like bodies in the cytoplasm (see insert). In the matrix surrounding the capillary, a very large mast cell-like cell with densely filled, homogeneous inclusions is visible (ic). Scale bars: (A) $25 \mu \mathrm{m}$, (B) $2 \mu \mathrm{m}$, (C) $500 \mathrm{~nm}$, (D) $3 \mu \mathrm{m}$.
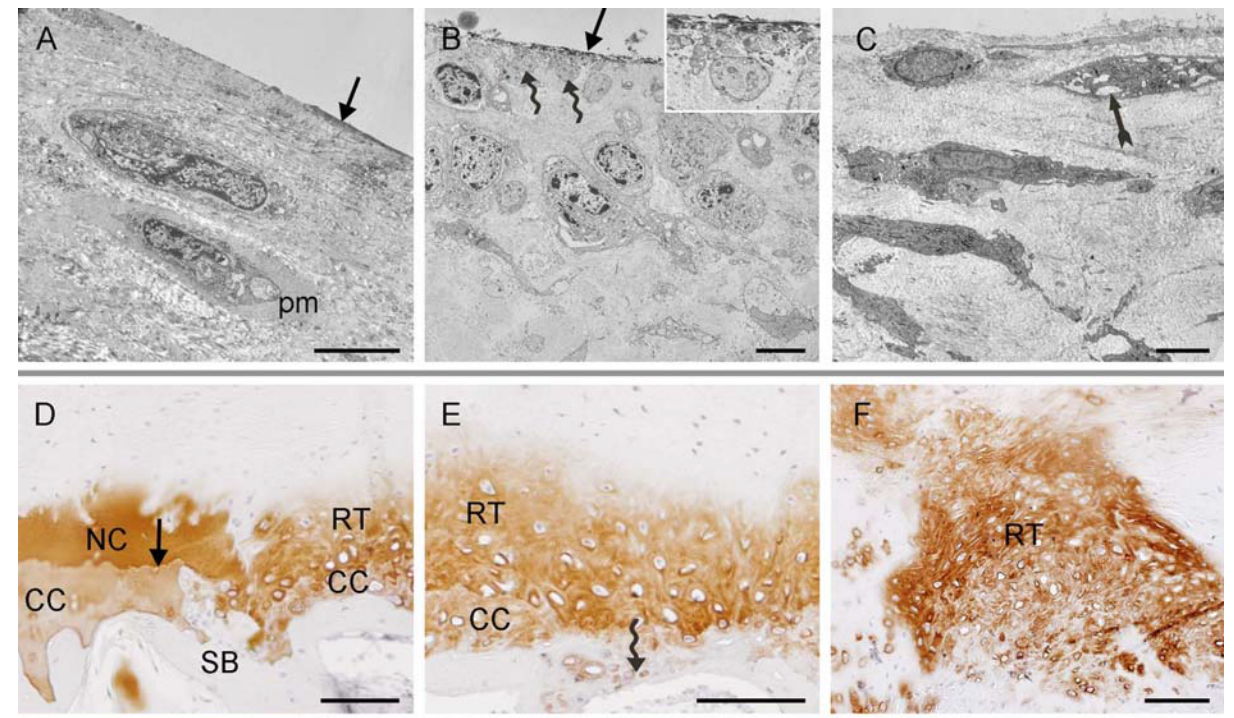

E
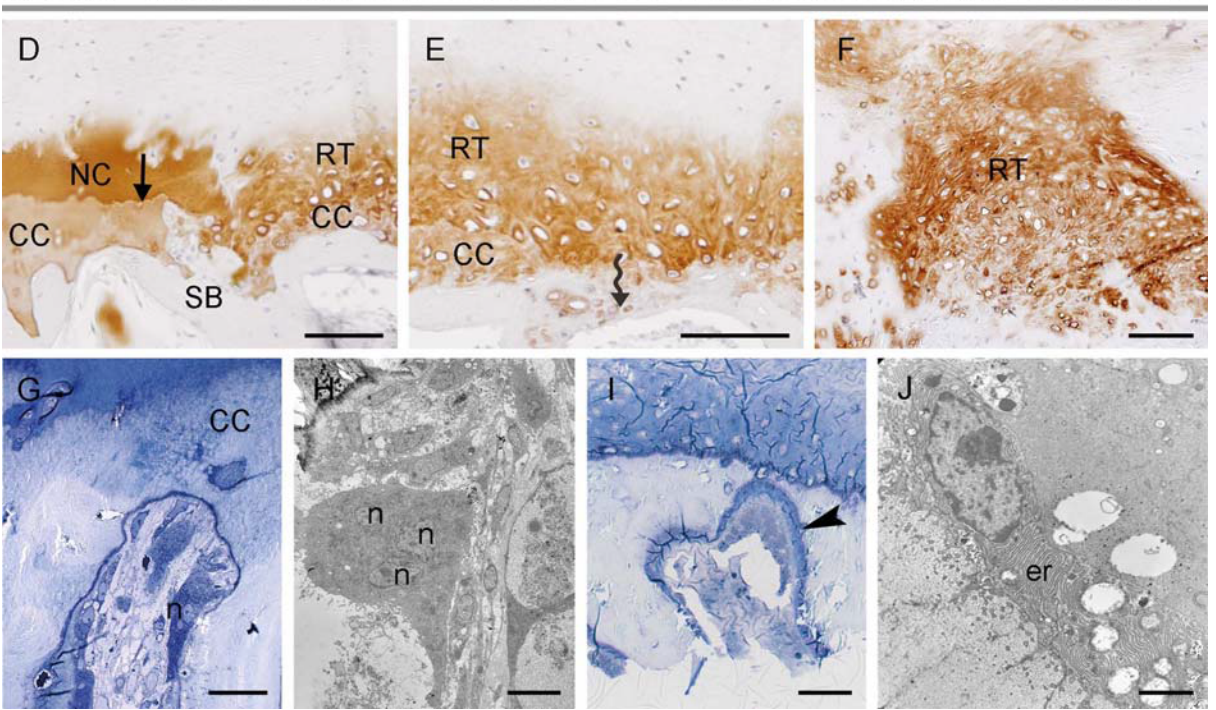

Figure 7: Upper and lower boundary of the RT. A-C: TEM images of the surface of the RT in treated defect (A, B) and the control (C). A: Elongated chondrocytes surrounded by a pericellular matrix ( $\mathrm{pm}$ ) are arranged parallel to the joint surface. The cells are surrounded by dense ECM, which is covered by a thin layer of electron-dense matrix (arrow). B: The cells at the surface extend cell extensions (wavy arrows and insert) and lack a pericellular matrix but are also embedded in dense matrix covered by dark material (arrow). C: Polygonal cells with many cell extensions and extended ER (arrow) are embedded in fibrous matrix. No electron dense material is visible at the surface. D-F: Immunohistochemical reactions against collagen type II in the deep region of transplanted defect show local differences of RT, CC and SB. D: Residual, homogeneous and intensively stained NC above a homogeneous, brighter-staining CC and unstained SB. The arrow indicates the tide mark. Adjacent to this there is collagen type II positive but somewhat fibrous RT. The underlying CC is hardly distinguishable from the RT but slightly brighter. A similar situation is also visible at the left side in E. E: In the middle of the image the trabeculae of the bone are very thin and the partially decalcified cartilage extends down to the bone lacuna of the subchondral bone (wavy arrow). F: Strong staining of the RT adjacent to a perforation of the SB. G,I: Toluidine blue and H,J: TEM images of cellular activity in the SB in transplanted defects. G, H: Osteoclasts with several Nuclei ( $\mathrm{n}$ ) are resorbing the SB such that the bone marrow lacuna penetrates the CC. I, J: Osteoblasts with abundant ER (er) deposit osteoid (arrowhead). Images A, B, D, E, I and $\mathrm{J}$ were taken form cartilage defect treated with a double sandwich-like layer of a hyaluronan web and a collagen type I/III fleece, $\mathrm{C}$ from the control defect without transplant, $\mathrm{F}$ from the defect treated with a single collagen type I/III fleece layer and $\mathrm{G}, \mathrm{H}$ with chondrocytes on a single hyaluronan web layer. Scale bars: (A-C) $5 \mu \mathrm{m}$, (D-F) $100 \mu \mathrm{m},(\mathrm{G}) 30 \mu \mathrm{m},(\mathrm{H}) 10 \mu \mathrm{m}$, (I) $50 \mu \mathrm{m},(\mathrm{J}) 2 \mu \mathrm{m}$. 
Citation: Sylvia N, Dirk B, Brigitte T, Igor P, Christof M, et al. (2013) (Ultra) Structural Details of Cells and Matrix during Early Cartilage Regeneration after Chondrocyte Implantation in the Equine Knee Joint. J Tissue Sci Eng S2: 004. doi:10.4172/2157-7552.S2-004

oriented alongside the collagen bundles (Figure 5C). Adapted to the cell shape, the nuclei had an elongated, curved or irregular outline. The intracellular features such as intermediary filament-bundles around the nuclei and lipid droplets became rare. Instead, the endoplasmic reticulum was especially expressed and sometimes dilated (Figure 6B); the number of mitochondria increased and abundant small vesicles (caveolae) were formed at the cell membrane (Figure 6C). Strands of microfilaments (actin) stretched especially into the cell projections, anchored locally to the cell membrane and formed focal junctions to the ECM (Figure 6C). Sometimes individual collagen fibrils penetrated the cell in deep invaginations of the membrane (Figure 6C). The matrix surrounding the cells was generally fibrous but sometimes a very thin layer of granular material was present (Figure 6B).

In deeper regions of fibrous RT, vascular-like structures were present, especially in the centre of the defect (Figure 6A and D). In the area around those structures, large cells containing numerous large vesicles with dense granular content were identified as mast cells.

The same morphological characteristics were found in the fibrous $\mathrm{RT}$ of the double layer transplanted defect (Figure $2 \mathrm{H}-\mathrm{J}$ ) and also in the tissue of the control (Figure 2M-O). In the latter, though, the ECM was less dense and more irregular; the cells were more ramified with many cell processes, exhibited an especially dark cytoplasm, and often showed a partially extended ER with bright lumen (Figure 2O).

\section{Surface of the repair tissue}

In all defects (treated with one or two transplant layers), the surface of the RT was generally straight, smooth and well delimited from the synovial space by ECM. Although the RT was fibrous in the upper region of the defect, the outermost layer (about $20 \mu \mathrm{m}$ ) showed local similarities to the NC surface. Elliptic cells were surrounded by a pericellular matrix and embedded in a partially masked collagen matrix (Figure 7A). Both cells and collagen fibres were oriented parallel to the surface. A fine layer of electron-dense, granular matrix of about $80 \mathrm{~nm}$ covered the superficial margin over large distances and seemed to lubricate the surface (Figure 7A and B). At other sites, the cell morphology was more polygonal or fibroblast-like and embedded between more or less masked collagen strands (Figure 5A and 7B). The control resembled the latter situation but the matrix and the cells were more irregular, bore numerous large and small cell processes and sometimes contained an extended endoplasmic reticulum (Figure 7C).

\section{Calcified Cartilage (CC) and Subchondral Bone (SB)}

During defect preparation, Calcified Cartilage (CC) was not removed but only superficially scratched. Consequently, the tide mark forming the upper margin of the CC should have been partially interrupted. Three months after transplantation, the CC and parts of the tide mark were still present in most sites but showed a different degree of degradation.

Completely unchanged CC was found at sites where the tide mark was intact and some NC remained (Figure 7D). In regions where the tide mark was missing, the CC had an irregular upper outline and a slightly inhomogeneous consistency. This probably resulted from endogenous decalcification and degradation of proteoglycans during the three-month regeneration of the defect (Figure 7D and E). Especially in the centre of the defect, the trabeculae of the subchondral bone also showed signs of degradation and were very thin or missing. At those sites the adjacent tissue (RT or endogenous decalcified cartilage) directly flanked the bone marrow cavities (Figure 7E). In some cases the RT close to the fenestrations was strongly positive for collagen type II (Figure 7F). The situations described above did not form a continuous transition from the periphery to the centre, but were recorded locally along the SP. Nevertheless, the CC and the RT had an intense contact with each other and a continuous transition at all sites.

In the Subchondral Bone (SB), osteoclasts advanced the medullar space towards the CC (Figure 7G and $\mathrm{H}$ ). We also recorded osteoblasts, which deposited new osteoid (Figure 7I and J). Generally, the bone level was very irregular and either above or below the original level (Figure 2C).

\section{Discussion}

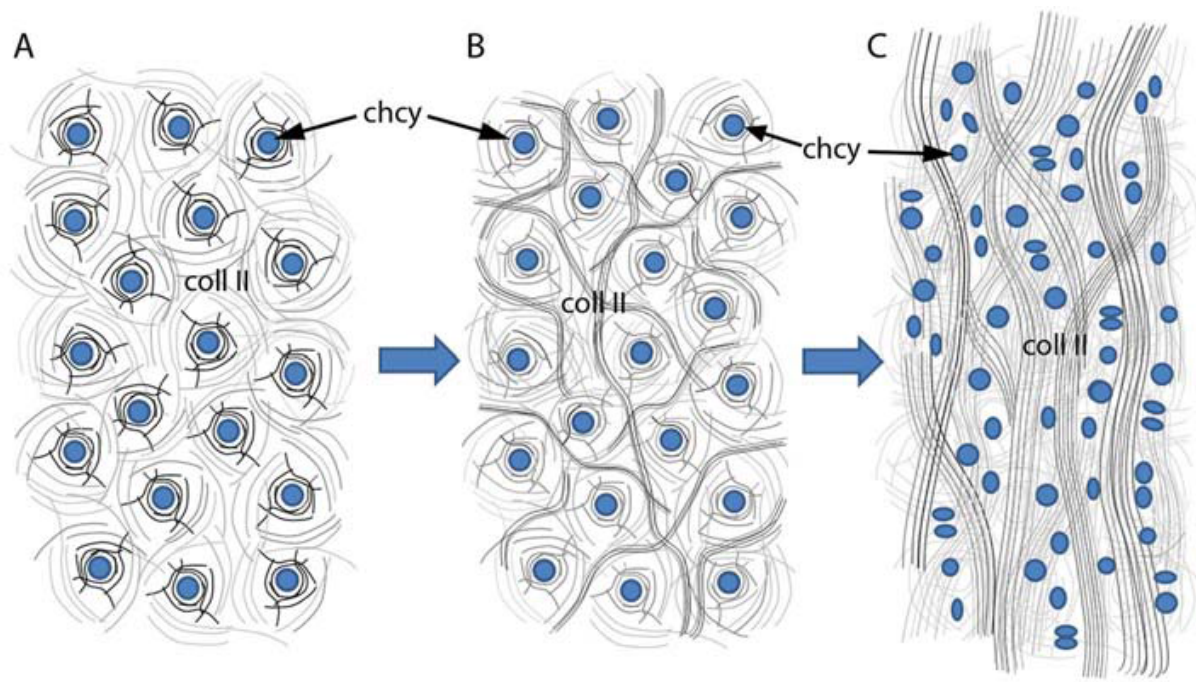

Figure 8: Hypothesis on the development and maturation of the collagen type II (coll II) arrangement in the RT. A: Initially, Chondrocytes (chcy) secrete collagen type II into their direct surroundings. B: The fibrils continuously form a fibre network (illustration corresponds to Figure 3E). C: With increasing collagen type II secretion, vertical fibre strands arrange and make up the bulk of the ECM fibres (illustration corresponds to Figure 3A). The previous network (of stage B) remains at least in parts but becomes quantitatively insignificant. 
The present study was designed to verify the horse as a suitable animal model for MACT transplants using soft scaffold materials as is done in humans. During this pilot study we asked the additional question of how tissue develops from the very different transplant materials at the early postoperative time point of three months.

\section{Horse model}

Despite the similarity of joint dimension as well as cartilage thickness, morphology, physiology and pathology in humans and the horse there are, of course, differences in the anatomy and the demands put on the knee of the two species [4-13]. Although the horse has a higher body weight, the load on the cartilage is spread over 4 legs $(60 \%$ on the forelegs) rather than two and loading on the cartilage appears comparable between the two species. However, it was necessary to test whether the cells and the soft carrier materials can endure the loading conditions in the equine joint [14]. In addition, in previous studies, human as well as equine chondrocytes showed very different cell distribution on the scaffolds depending on the material density [6]. Therefore, we also aimed to test if the transplants are able to give rise to a defect-filling RT. Three months after implantation, all transplanttreated defects were filled with RT while the control defect was almost empty. This indicates that the transplants remained in the defect and a tissue was able to develop from both the loose and dense transplant type. Nonetheless, the defect filled with a double-layer transplant was less filled with RT than those treated with a single-layer transplant only. This suggests that the upper transplant was pulled out of the defect during the postoperative period. Only the lower transplant, the soft hyaluronan web, remained compressed by the upper one and no longer completely filled the defect after the upper layer was lost. These results show that the soft transplants withstand the mechanical loading conditions in the equine joint, but still are at risk of being displaced if they are subjected to shear force on the joint surface. The surgery and the postoperative follow-up care were uncomplicated, showing that the surgical procedure can also be considered suitable for horses from the technical and ethical points of view.

\section{Peripheral differentiation of the repair tissue (RT)}

In the horse cartilage, differentiation of the RT (collagen type II and homogeneous toluidine blue $\mathrm{O}$ staining) clearly appeared in the periphery adjacent to the $\mathrm{NC}$ and in the depth of the defect. This is a common feature in cartilage regeneration and according to the present observations, it can be related to either mechanical reasons or the influence of NC; at the interface to the $\mathrm{NC}$, mechanical loading is probably more intense than in the middle, where the RT is somewhat collapsed (only $1 / 2$ to $3 / 4$ the height of the RT in the periphery) [15-17]. Dynamic mechanical load is one of the key stimuli of differentiation, in vitro as well as in vivo, and influences matrix composition, collagen structure and dimension [18-22].

Apart from the mechanical loading conditions, the proximity of the differentiated RT to the NC makes it probable that the latter influenced the development of tissue in the defect. As known from experimental studies, differentiated chondrocytes may have a "teaching effect" on other cells such as dedifferentiated chondrocytes or stem cells (MSCs, ASCs); the trigger proceeds via cytokines or direct cell contact [2326]. It is possible that the chondrocytes in the bordering NC released cytokines which triggered the implanted chondrocytes in the defect to differentiate.

\section{Organisation of the hyaline-like matrix}

As mentioned above, the matrix of the peripheral RT stained strongly for collagen type II, which is the most common criterion for cartilage differentiation [17,27]. Another important but frequently disregarded aspect of the functionality of articular cartilage is the matrix architecture. Typical for articular cartilage is collagen arranged in vertical arcades that consist of leaf-like or tube-like substructures [28-30]. Vertical arrangement of the collagen type II fibres was also observed in the differentiated part of the RT. The arrangement apparently developed from a collagen type II-network, which was formed directly around the cells (Figure 8A). This network becomes tangential and finally vertical with increasing collagen type II fibre deposition. It seems likely that the collagen type II-network around the chondrocytes remains, at least temporarily, but becomes overlaid by an increasing amount of vertically arranged fibres (Figure $8 \mathrm{~B}$ and $\mathrm{C}$ ). Tangential fibrils crossing the main fibre direction are also known from $\mathrm{NC}$ and were previously identified using scanning and transmission electron microscopy [31]. However, a development of the collagen architecture from network-like to vertical has, to our knowledge, never been described before, neither in regeneration nor developmental studies. Rather, ontogenetic cartilage maturation is described as replacement of a horizontally oriented collagen (parallel to the joint surface) by a vertical one $[32,33]$. Why such a process as proposed in Figure 8 has not been described in earlier studies could be due to the following reasons: 1) The network is too fine to be determined with normal paraffin histology (here we used deplastified $1 \mu$-resin sections) and too large to be assessed in (70 nm-thick) ultrathin sections using transmission electron microscopy, and/or 2) a fine fibre network is difficult to distinguish inside pre-existing tissue such as that present during the matrix maturation of postembryonic development. The present study provides first insights into a possible matrix organisation in developing RT. Nonetheless, further studies are required to collect more information about this process. One interesting aspect is a possible difference in matrix deposition and maturation depending on whether collagen type II replaces a pre-existing matrix or is deposited in an empty space.

Despite the large correspondence of the differentiated RT with mature cartilage, the organisation of RT still differs from the mature tissue in terms of high cellularity, lower matrix density, lower proteoglycan content as well as the lack or rare appearance of a pericellular and territorial matrix. All those features are, however, typical for immature articular cartilage and support the suggestion that this tissue is in the process of maturation [34-37]. This further means that collagen type II is the earliest morphologically distinctive sign of RT-differentiation, preceding proteoglycan deposition and matrix compartmentalisation.

\section{Calcified cartilage (CC) and subchondral bone (SB)}

The role of CC in defect healing in full-thickness defects in in vivo studies is controversial. In the horse, CC was reported to hamper adhesion of RT after microfracture [38]. In dogs, either good or variable adhesion to CC was found after different implantation methods (cells, scaffolds, periost) without SB perforation $[17,39]$. In the present study, the $\mathrm{CC}$ was not removed but superficially scratched during surgery. Three months after implantation, the CC was locally decalcified and the transition of the RT to the CC continuous. Likewise, a stress-line was prevented and the interface between the CC and the RT was strengthened. The present observations reveal that partial endogenous degradation of CC may contribute to a good bonding of RT to the SP.

Locally, the SB displayed fenestration in the third postoperative month, connecting the defect area to bone marrow. Since no bleeding was observed during surgery, micro-perforation during 
Citation: Sylvia N, Dirk B, Brigitte T, Igor P, Christof M, et al. (2013) (Ultra) Structural Details of Cells and Matrix during Early Cartilage Regeneration after Chondrocyte Implantation in the Equine Knee Joint. J Tissue Sci Eng S2: 004. doi:10.4172/2157-7552.S2-004

Page 9 of 10

defect preparation is unlikely. Instead, the perforations apparently develop postoperatively through intrinsic mechanisms such as the decalcification of the CC and the resorption of the SB by osteoclasts found in the trabecular system. In previous studies, postoperative bone remodelling has been attributed to endogenous cells reacting to the degradation products of the scaffold or to implanted cells [16,39]. In a further scaffold-implantation study, untreated control defects also revealed subchondral remodelling, indicating that remodelling was not related to the implanted material [40]. The detailed histological and ultrastructural findings of this study also showed no indication that SB-remodelling is related to the implanted material (e.g. foreign body reaction or inflammation). Rather, the SB remodelling may well be related to a reduced loading in those particular regions. Previous immobilisation studies support this suggestion because they also reported an association between SB-remodelling and mechanical demand $[41,42]$. Osteoblasts found in some areas at the base of the SP suggest counteracting bone reconstruction, which probably explains the alteration of bone surface levels found in this and other studies $[7,43]$.

\section{Clinical relevance \& limitations}

The detailed morphological analyses presented in this study could give insights into the clinical relevant topics of cartilage repair, tissue differentiation and integration. Complete defect filling seems to support repair tissue differentiation indicating that transplants should be at equal level as the defect and stiff enough to keep the shape under the demand of movement. In this study defects differentiation started at the periphery and was probably positively influenced by vital chondrocytes in the adjacent native cartilage. This implicates that defect preparation for removal of degraded cartilage is important, but has to be done with care to prevent further cell damage. The study further contributes to the controversial discussion on the function of calcified cartilage in repair tissue integration demonstrating that good bonding is possible even with calcified cartilage. Furthermore, morphological details of matrix characteristic allowed describing a sequence of matrix deposition which may possibly be specific to the regeneration process. This raised the question whether the sequence of matrix maturation is essential for cartilage tissue differentiation, and whether this process could be supported by adequate biomaterial architecture.

The study is limited by the low sample size in this pilot study. Therefore no statistically relevant difference could be observed between transplant types. The focus of this study was the in vivo development of the transplants in the course of the first months after implantation. However data on the long term outcome may contribute further relevant information in the future.

\section{Conclusion}

Morphological investigations revealed that differentiation starts at the periphery of the defects and that collagen type II is the first morphologically detectable ECM component. We propose a sequence of collagen type II fibre deposition from network-like to vertical. Remodelling of CC was found to lead to good bonding of RT to the SP. The complication-free surgery and post-operative follow-up care, good defect filling and initial differentiation after three months confirm that the horse is an appropriate animal model. Long-term experiments with a higher number of defects treated with MACT soft scaffolds would be helpful to obtain further information about defect development and differences between scaffold types.

\section{Acknowledgements}

The study was in part supported by a Lorenz-Boehler grant and the EU-Project FA735A0104 titled "A Systematic Approach to Tissue Engineering Processes and Products (STEPS)".The authors would like to thank Dr. Guenter Resch from the IMP-IMBA-GMI Electron Microscopy Facility in Vienna and the "Cell Imaging and Ultrastructure Research Unit" CIUS for providing the equipment for the electron and light microscopic investigations, and Tamara Jagersberger from the Department of Traumatology of the Medical University of Vienna for her support in cell culturing. Furthermore, we would like to acknowledge Mag. Norbert Cyran, Dr. Janek von Byern and Prof. Heinz Redl for critically reading the manuscript. This study is part of the $\mathrm{PhD}$ Thesis of Sylvia Nürnberger.

\section{References}

1. Marlovits S, Nurnberger S, Kolonja A, Singer P, Zeller P, Mandl I (2004) Matrixgekoppelte autologe Knorpelzelltransplantation - Biomaterialien als Trägersubstanzen / Biologische Eigenschaften. Trauma und Berufskrankheit 6: 314-323.

2. Hunziker EB (2003) Tissue engineering of bone and cartilage. From the preclinical model to the patient. Novartis Found Symp 249: 70-78.

3. Hunziker EB (1991) Articular Cartilage Structure in Humans and Experimental Animals. In: Articular Cartilage and Osteoarthritis. editors: Kuettner KE Schleyerbach R, Peyron JG et al. New York: Raven Press 183-200.

4. Frisbie DD, Cross MW, Mcllwraith CW (2006) A comparative study of articular cartilage thickness in the stifle of animal species used in human pre-clinical studies compared to articular cartilage thickness in the human knee. Vet Comp Orthop Traumatol 19: 142-146.

5. Ahern BJ, Parvizi J, Boston R, Schafer TP (2013) Preclinical animal models in single site cartilage defect testing: a systematic review. Osteoarthritis Cartilage 17: 705-713.

6. Nürnberger S, Meyer C, Ponomarev I, Barnewitz D, Resinger C, et al. (2012) Equine articular chondrocytes on MACT-scaffolds for cartilage defect treatment. Anat Histol, Embryol 42: 332-343

7. Barnewitz D, Endres M, Kruger I, Becker A, Zimmermann J, et al. (2006) Treatment of articular cartilage defects in horses with polymer-based cartilage tissue engineering grafts. Biomaterials 27: 2882-2889.

8. Barnewitz D, Meyer C, Ponomarev I, Karakine E, Marlovits S, et al. (2008) New diagnostic and therapeutical approaches of cartilage diseases. Proceedings $10^{\text {th }}$ International Congress of World Equine Veterinary Association 2008: 608-619.

9. Frisbie DD, Oxford JT, Southwood L, Trotter GW, Rodkey WG, et al. (2003) Early events in cartilage repair after subchondral bone microfracture. Clin Orthop Relat Res 407: 215-227.

10. Buckwalter JA, Hunziker EB, Rosenberg L, Coutts RD, Adams M, et al. (1988) Articular Cartilage: Composition and Structure. In: Injury and Repair of the Musculoskeletal Soft Tissues. editors: Woo SS-Y, Buckwalter JA. Park Ridge Illinois: American Academy of Orthopaedic Surgeons 405-25.

11. Noonan KJ, Hunziker E, Nessler J, Buckwalter JA (1998) Changes in cell, matrix compartment, and fibrillar collagen volumes between growth-plate zones. J Orthop Res 16: 505-508.

12. Marlovits S, Tichy B, Truppe M, Gruber D, Schlegel W (2003) Collagen expression in tissue engineered cartilage of aged human articular chondrocytes in a rotating bioreactor. Int J Artif Organs 26: 319-330.

13. Mcllwraith CW, Frisbie DD, Kawacak CE (2013) The horse as a model of naturally occurring osteoarthritis. Bone Joint Res 1: 297-309.

14. Nürnberger S, Marlovits S (2006) Electronmicroscopic comparison of HyalograftC versus Chondrogide ${ }^{\otimes} / \mathrm{MACl}^{\otimes}$. In: Basic science, clinical repair and reconstruction of articular cartilage defects: current status and prospects. editors: Zanasi S, Brittberg M, Nehrer S et al. Bologna: Timeo 695-704.

15. Breinan HA, Minas T, Barone L, Tubo R, Hsu HP, et al. (1998) Histological evaluation of the course of healing of canine articular cartilage defects treated with cultivated autologous chondrocytes. Tissue Eng 41: 101-114.

16. Breinan HA, Martin SD, Hsu HP, Spector M (2000) Healing of canine articula cartilage defects treated with microfracture, a type-II collagen matrix, or cultured autologous chondrocytes. J Orthop Res 18: 781-789. 
Citation: Sylvia N, Dirk B, Brigitte T, Igor P, Christof M, et al. (2013) (Ultra) Structural Details of Cells and Matrix during Early Cartilage Regeneration after Chondrocyte Implantation in the Equine Knee Joint. J Tissue Sci Eng S2: 004. doi:10.4172/2157-7552.S2-004

17. Breinan HA, Minas T, Hsu HP, Nehrer S, Shortkroff S, et al. (2001) Autologous chondrocyte implantation in a canine model: change in composition of reparative tissue with time. J Orthop Res 19: 482-492.

18. Frisbie DD, Al Sobayil F, Billinghurst RC, Kawcak CE, Mcllwraith CW (2008) Changes in synovial fluid and serum biomarkers with exercise and early osteoarthritis in horses. Osteoarthritis Cartilage 16: 1196-1204.

19. Kawanishi M, Oura A, Furukawa K, Fukubayashi T, Nakamura K, et al. (2007) Redifferentiation of dedifferentiated bovine articular chondrocytes enhanced by cyclic hydrostatic pressure under a gas-controlled system. Tissue Eng 13 957-964

20. Skiöldebrand E, Ekman S, Heinegård D, Hultenby K (2010) Ultrastructura immunolocalization of cartilage oligomeric matrix protein (COMP) in the articular cartilage on the equine third carpal bone in trained and untrained horses. Res Vet Sci 88: 251-257.

21. Tagil M, Aspenberg $P$ (1999) Cartilage induction by controlled mechanical stimulation in vivo. J Orthop Res 17: 200-204.

22. Smith RL, Lindsey DP, Dhulipala L, Harris AHS, Goodman SB, et al. (2011) Effects of intermittent hydrostatic pressure and BMP-2 on human chondrocyte metabolism in vitro. J Orthop Res 29: 361-368.

23. Hendriks J, Riesle J, van Blitterswijk CA (2007) Co-culture in cartilage tissue engineering. J Tissue Eng Regen Med 1: 170-178.

24. Acharya C, Adesida A, Zajac P, Mumme M, Riesle J, et al. (2012) Enhanced chondrocyte proliferation and mesenchymal stromal cells chondrogenesis in coculture pellets mediate improved cartilage formation. J Cell Physiol 227: 8897.

25. Gan L, Kandel RA (2007) In vitro cartilage tissue formation by Co-culture of primary and passaged chondrocytes. Tissue Eng 13: 831-842.

26. Hildner F, Peterbauer A, Wolbank S, Nurnberger S, Marlovits S, et al. (2010) FGF-2 abolishes the chondrogenic effect of combined BMP-6 and TGF-beta in human adipose derived stem cells. J Biomed Mater Res A 94: 978-987.

27. Nugent AE, Reiter DA, Fishbein KW, McBurney DL, Murray T, et al. (2010) Characterization of ex vivo-generated bovine and human cartilage by immunohistochemical, biochemical, and magnetic resonance imaging analyses. Tissue Eng Part A 16: 2183-2196.

28. Benninghoff A (1925) Form und Bau der Gelenkknorpel in ihren Beziehungen und Funktionen. Zweiter Teil: Der Feinbau des Gelenkknorpels in seinen Beziehungen zur Funktion. Z Zellforsch 2: 783-862

29. Jeffery AK, Blunn GW, Archer CW, Bentley G (1991) Three-dimensional collagen architecture in bovine articular cartilage. J Bone Joint Surg $\mathrm{Br} 73$ : 795-801.

30. AP Gwynn I, Wade S, Kaab MJ, Owen GR, Richards RG (2000) Freezesubstitution of rabbit tibial articular cartilage reveals that radial zone collagen fibres are tubules. J Microsc 197: 159-172.
31. AP Gwynn I, Wade S, Ito K, Richards RG (2002) Novel aspects to the structure of rabbit articular cartilage. Eur Cell Mater 4: 18-29.

32. Archer CW, Dowthwaite GP, Francis-West P (2003) Development of synovia joints. Birth Defects Res C Embryo Today 69: 144-155.

33. Rieppo J, Hyttinen MM, Halmesmaki E, Ruotsalainen $H$, Vasara A, et al. (2009) Changes in spatial collagen content and collagen network architecture in porcine articular cartilage during growth and maturation. Osteoarthritis Cartilage 17: 448-455.

34. Khan IM, Evans SL, Young RD, Blain EJ, Quantock AJ, et al. (2011) Fibroblast growth factor 2 and transforming growth factor beta1 induce precocious maturation of articular cartilage. Arthritis Rheum 63: 3417-3427.

35. Vincent T, Hermansson M, Bolton M, Wait R, Saklatvala J (2002) Basic FGF mediates an immediate response of articular cartilage to mechanical injury. Proc Natl Acad Sci U S A 99: 8259-8264.

36. Vincent TL, Hermansson MA, Hansen UN, Amis AA, Saklatvala J (2004) Basic fibroblast growth factor mediates transduction of mechanical signals when articular cartilage is loaded. Arthritis Rheum 50: 526-533.

37. Hunziker EB, Kapfinger E, Geiss J (2007) The structural architecture of adult mammalian articular cartilage evolves by a synchronized process of tissue resorption and neoformation during postnatal development. Osteoarthritis Cartilage 15: 403-413.

38. Frisbie DD, Morisset S, Ho CP, Rodkey WG, Steadman JR, et al. (2006) Effects of calcified cartilage on healing of chondral defects treated with microfracture in horses. Am J Sports Med 34: 1824-1831.

39. Nehrer S, Breinan HA, Ramappa A, Hsu HP, Minas T, et al. (1998) Chondrocyteseeded collagen matrices implanted in a chondral defect in a canine model. Biomaterials 19: 2313-2328.

40. Vasara Al, Hyttinen MM, Lammi MJ, Lammi PE, Langsjo TK, et al. (2004) Subchondral bone reaction associated with chondral defect and attempted cartilage repair in goats. Calcif Tissue Int 74: 107-114.

41. Jozsa L, Reffy A, Jarvinen M, Kannus P, Lehto M, et al. (1988) Cortical and trabecular osteopenia after immobilization. A quantitative histological study of the rat knee. Int Orthop 12: 169-172.

42. van Harreveld PD, Lillich JD, Kawcak CE, Turner AS, Norrdin RW (2002) Effects of immobilization followed by remobilization on minera density, histomorphometric features, and formation of the bones of the metacarpophalangeal joint in horses. Am J Vet Res 63: 276-281.

43. Schneider U, Gotterbarm H, Voss H, Stofft E, Graeve T, et al. (2006) Comparative study of traditional ACT and a novel collagen-gel based matrix augmented with autologous chondrocytes in a minipig model. In: Basic science clinical repair and reconstruction of articular cartilage defects: current status and prospects. editors: Zanasi S, Brittberg M, Nehrer S et al. Bologna: Timeo 743-53. 